\title{
CD36 Abnormality and Impaired Myocardial Long-Chain Fatty Acid Uptake in Patients With Hypertrophic Cardiomyopathy
}

\author{
Fumio Okamoto, MD; Takao Tanaka, MD; Koichi Sohmiya, MD; Keishiro Kawamura, MD
}

\begin{abstract}
Some patients with hypertrophic cardiomyopathy (HCM) demonstrate abnormal myocardial long-chain fatty acid (LCFA) metabolism. However, the exact mechanism involved is unknown. Recently, it was proposed that myocardial cells take up LCFAs via a specific mechanism, in which the CD36 molecule has been implicated as a possible candidate molecule. In addition, a high prevalence of CD36 deficiency was also found in a small number of HCM patients. Accordingly, the investigation of abnormality of the CD36 molecule in a large number of HCM patients may be useful in finding the possible cause of HCM. Moreover, the analysis of myocardial LCFA uptake in patients with molecular abnormalities may be helpful in understanding the possible function of this molecule. In this study, in order to discover the relationship between HCM and the CD36 molecular abnormality, the expression level of platelet CD36 and CD36 cDNA in $55 \mathrm{HCM}$ patients was analyzed. Twelve patients showed negligible $(<5 \%)$ CD36 expression on their platelets. Among them, one was found to be homozygous for the C-478 T substitution and 6 were heterozygous for the C-478 T substitution. In 9patients, CD36 was expressed by less than $50 \%$ of the platelets. One of them was found to be heterozygous for the C-478 T substitution. Two other patients were also found to be heterozygous for this point mutation, although their platelets expressed CD36. Thus, 23 out of $55(41.8 \%)$ HCM patients had negligible $(<5 \%)$ or reduced $(<50 \%)$ levels of CD36 expression on platelets, or had a point mutation of CD36 cDNA. These $55 \mathrm{HCM}$ patients were also evaluated with myocardial scintigraphy both for LCFA uptake and perfusion, which showed a moderate to severe discrepancy between myocardial LCFA accumulation and myocardial perfusion in 95.5\% of the patients (21/23). On the other hand, $70 \%$ of the patients with normal (>90\%) CD36 expression (14/20) did not show any severe discrepancies between myocardial LCFA accumulation and myocardial perfusion. These data could suggest that abnormal myocardial LCFA metabolism seen in HCM patients may be related to abnormality of the CD36 molecule, and that abnormalities of this molecule may be linked to the cause of some types of HCM. (Jpn Circ J 1998; 62: 499-504)
\end{abstract}

Key Words: Long-chain fatty acid; CD36 molecule; Hypertrophic cardiomyopathy

A lthough long-chain fatty acids (LCFAs) are a major energy source for the heart, the molecular mechanisms of myocardial LCFA uptake have not been well established. We isolated a myocardial LCFA transporter from bovine hearts and its amino acid sequence showed homology to human CD36 or rat FAT (as referred to by Abumurad et $\left.\mathrm{al}^{1}\right)^{2}$.

CD36 [also known as platelet glycoprotein IV (GP IV) and GP IIIb] is the fourth major glycoprotein expressed on the platelet surface and its deficiency is found in about 3\% of the Japanese population ${ }^{3}$ and about $0.3 \%$ of the US population. Although multifunction has been proposed for the CD36 molecule, individuals with CD36 deficiency are reported to be apparently healthy5 Thus, the precise pathophysiology of the abnormality of the CD36 molecule is still unclear.

On the basis of our finding that a molecule contributing to myocardial LCFA uptake showed homology to human

(Received September 25,1997; revised manuscript received February 17, 1998; accepted February 20, 1998)

Third Division, Department of Internal Medicine, Osaka Medical College, Takatsuki, Osaka, Japan

Mailing address: Takao Tanaka, MD, Third Division, Department of Internal Medicine, Osaka Medical College, Takatsuki, Osaka, Japan 569
CD36, we screened an abnormality of the CD36 molecule in individuals whose myocardial scintigrams demonstrated abnormal myocardial LCFA accumulation. We recently encountered individuals with complete absence of myocardial LCFA accumulation by scintigraphy in whom the CD36 molecule was not expressed on either the platelets or the monocytes and their CD36 cDNA showed homozygous for the C-478 T substitution'-8 Accordingly, we believe that $\mathrm{CD} 36$ is a possible candidate molecule for myocardial LCFA uptake.

Some patients with hypertrophic cardiomyopathy (HCM) have been reported to show impaired myocardial LCFA uptake, although the exact mechanism involved is unknown. We previously reported that inhibition of myocardial LCFA transport also induced cardiac hypertrophy in rats 10 A possible relationship between a shift in myocardial substrate utilization and cardiac hypertrophy has been suggested!1 Accordingly, we hypothesized that the first step in LCFA metabolism, ie, LCFA uptake mechanism at myocardial cell membrane, might be related to impaired myocardial LCFA uptake in HCM, and that an abnormality of this mechanism might be linked to the etiology of HCM.

In order to test our hypothesis, we recently screened platelet and monocyte CD36 expression in patients with 


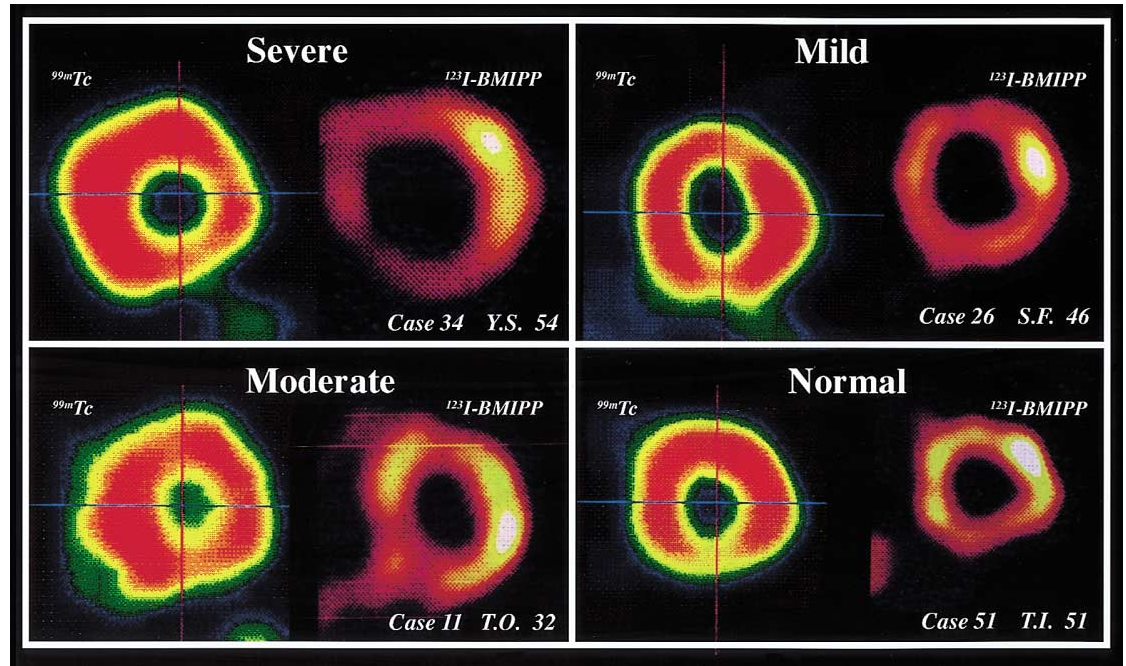

Fig 1. Representative cases for myocardial BMIPP accumulation.

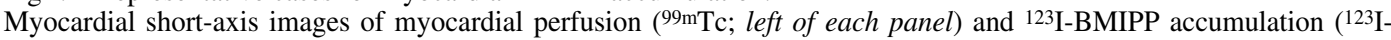
BMIPP; right of each panel) in patients with hypertrophic cardiomyopathy. Based on the discrepancy between myocardial BMIPP accumulation and flow tracer accumulation, the patients were divided into 5 groups; Normal = no discrepancy between BMIPP and flow tracer accumulation, Mild=mildly decreased BMIPP accumulation relative to flow tracer accumulation, Moderate $=$ moderately decreased BMIPP accumulation, Severe $=$ severely decreased BMIPP accumulation, and None $=$ no myocardial BMIPP accumulation despite apparently normal flow tracer accumulation. Case 34, scored as severe, myocardial BMIPP accumulation was apparently decreased at the hypertrophied interventricular septal region, in which myocardial perfusion was seemed to be normal. Case 11, scored as moderate, myocardial BMIPP accumulation at the interventricular septal and endomyocardial region was moderately reduced despite apparently normal myocardial perfusion at that region. Case 26, scored as mild, myocardial BMIPP accumulation was reduced especially at the endomyocardial region.

cardiac hypertrophy. In that preliminary study, we found that there was a high prevalence of CD36 deficiency in patients with HCM6 Knowledge of the abnormality of the CD36 molecule and the dynamics of myocardial LCFA uptake may be helpful in understanding the mechanism of abnormal myocardial LCFA metabolism in some HCM patients. Thus, in this study, we investigated the expression of the CD36 molecule on platelets and monocytes, and analyzed CD36 cDNA in patients with HCM, who were also evaluated for myocardial LCFA uptake and myocardial perfusion by scintigraphy.

\section{Methods}

\section{Patients}

Fifty-five HCM patients (44 men and 11 women with a mean age of $51.7 \pm 12.6$ years, range $20-82$ years) who were evaluated for both myocardial LCFA uptake and perfusion within 1 year were included in this study. The diagnosis of HCM was based on echocardiographic detection of an interventricular septum and/or posterior wall thickness greater than $1.3 \mathrm{~cm}$. All patients showed no left ventricular dilation and there was no evidence of another cardiac or systemic disease that could cause such hypertrophy.

Iodine-123 15(p-iodophenyl)-3-R,S-Methylpentadecanoic Acid (123I-BMIPP) and Tetrofosmin Technetium-99m ( $\left.{ }^{99 m} T c\right)$ or Thallium-201 Chloride $\left({ }^{201} \mathrm{Tl}\right)$ Myocardial Single-Photon Emission Computed Tomography (SPECT)

Myocardial LCFA uptake and myocardial perfusion were respectively evaluated using $123 \mathrm{I}-\mathrm{BMIPP}$ and $99 \mathrm{mTc}$ or ${ }^{201} \mathrm{Tl}$ at the Department of Radiology of Osaka Medical College Hospital. After an overnight fast, $111 \mathrm{MBq}$ of ${ }^{123} \mathrm{I}-$
BMIPP (Nihon Medi-Physics, Japan) was administered iv and SPECT imaging was carried out 20 min after injection. Myocardial perfusion studies were obtained at rest on another day after iv injection of $99 \mathrm{~m} \mathrm{Tc}(15 \mathrm{MBq})$ or ${ }^{201} \mathrm{Tl}$ (74 MBq). Myocardial perfusion imaging was obtained 30 min after ${ }^{99 \mathrm{~m}} \mathrm{Tc}$ or ${ }^{201} \mathrm{Tl}$ injection.

\section{Myocardial BMIPP Uptake and Myocardial Perfusion Analysis}

Both myocardial BMIPP uptake and perfusion scintigraphy were carried out at a mean interval of 3.0 months (1-12 months). In 28 patients, BMIPP uptake scintigraphy was performed at $1-12$ months (mean 3.1 \pm 3.1 months) before the myocardial perfusion study, whereas the remaining 27 patients underwent the perfusion study $1-10$ months (mean $2.9 \pm 3.9$ months) before the myocardial BMIPP uptake study.

Myocardial BMIPP accumulation was compared with myocardial perfusion ( ${ }^{99 \mathrm{~m}} \mathrm{Tc}$ or ${ }^{201} \mathrm{Tl}$ scintigraphy) on all slices obtained in the short-axis, horizontal, and vertical long-axis views. A good image quality was obtained with myocardial perfusion studies in all patients, whereas myocardial BMIPP images were relatively poor in patients with CD36 deficiency because of impaired or no myocardial accumulation of the radioisotope. However, no patient showed higher BMIPP accumulation than flow tracer accumulation ( ${ }^{99 \mathrm{~m} T c}$ or $\left.{ }^{201} \mathrm{Tl}\right)$ in all segments of these 3 views. Based on the discrepancy between myocardial BMIPP accumulation and flow tracer accumulation, the patients were divided into 5 groups: normal (no discrepancy between BMIPP and flow tracer accumulation); mild (mildly decreased BMIPP accumulation relative to flow tracer accumulation); moderate (moderately decreased 
Detection of the Pro90 Ser mutation in CD36 cDNA
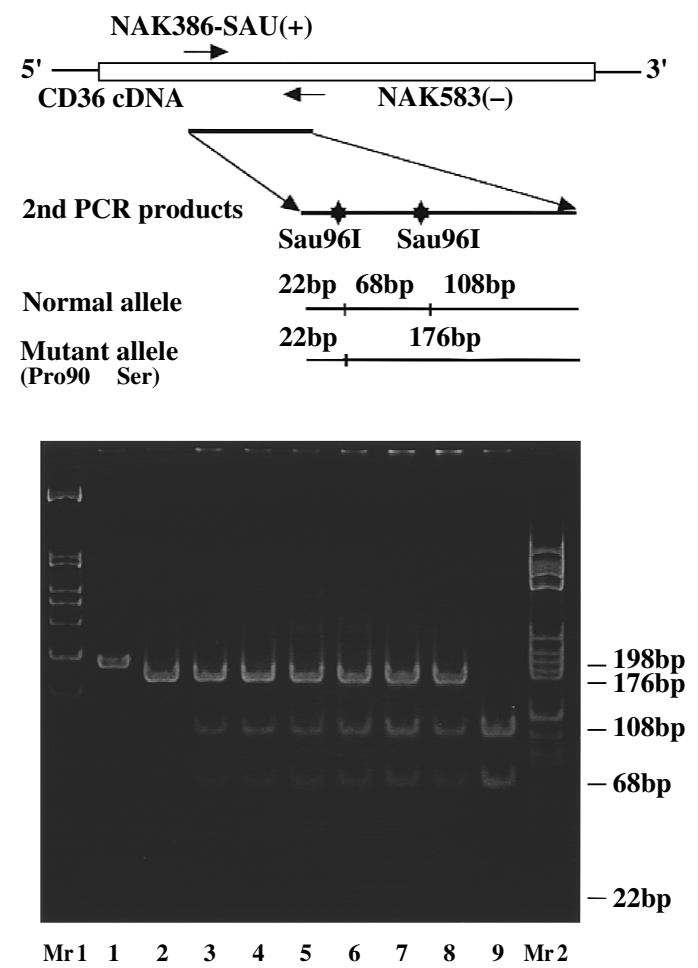

Fig 2. Detection of the C-478 T substitution in CD36 cDNA by RT-PCR followed by Sau96I digestion.

The schema shows the amplification of CD36 cDNA at the interested region and Sau96I digestion sites. A 198-bp fragment of CD36 cDNA (nt 386 to 583) was amplified and digested with Sau96I. This fragments contained a new Sau96I site at nt 408 generated by mismatched-PCR for internal standard. The photogram shown are for 1 wild-type, 6 heterozygotes for C-478 T substitution, and 1 homozygote for C-478 T substitution. The absence of the 198-bp band in all samples showed complete digestion by Sau96I. The C-478 T substitution led to loss of a Sau96I site at nt 476 . The C-478 form of CD36 cDNA is digested to 108-, 68-, and 22- (invisible) bp fragments, while the T-478 form of $C D 36$ cDNA is digested to 176- and 22- (invisible) bp fragments. Lane 1, 198-bp product from wild-type CD36 cDNA (without Sau96I digestion); lane 2, homozygote for the C-478 T substitution (case 1); lanes 3-8, heterozygotes for the C-478 T substitution (case 4, 11, 12, 31, 34, and 13); lane 9, Sau96I digestion of the 198-bp product from wild-type $C D 36$ cDNA. Mr 1 and Mr 2, molecular standards, HindIII digestion of $\lambda$ DNA and HinfII digestion of pBR322, respectively.

BMIPP accumulation); severe (severely decreased BMIPP accumulation); and none (no myocardial BMIPP accumulation despite apparently normal flow tracer accumulation). This arbitrary classification was determined visually for all slices in the 3 axis views. Fig 1 illustrates the representative cases according to our arbitrary classification.

\section{Preparation of Platelets and Monocytes and Flow Cytometry}

Heparinized blood was collected from an antecubital vein after informed consent was obtained. Collection of blood was performed within 1.5 years after the examination of myocardial scintigraphy. Platelet-rich plasma (PRP) and mononuclear cells (MNCs) were prepared as described previously?

PRP was diluted to $1 \times 10^{8} / \mathrm{ml}$ with phosphate-buffered

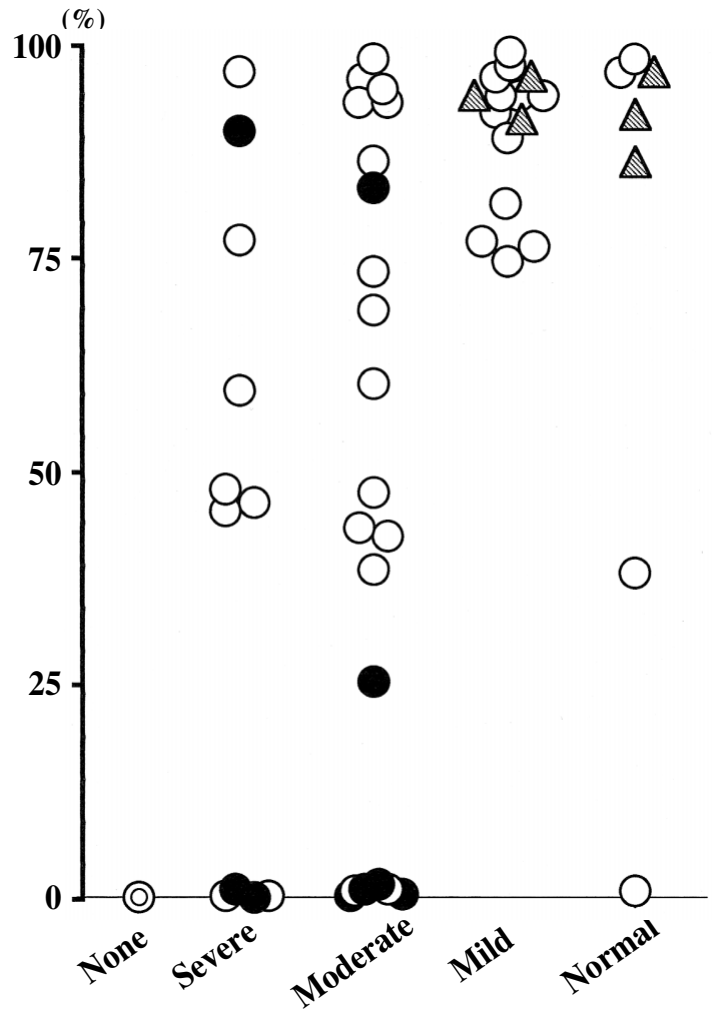

Fig 3. Relationship between myocardial BMIPP accumulation (arbitrary score) and the expression of CD36 on platelets.

Based on the discrepancy between myocardial BMIPP accumulation and flow tracer accumulation, the patients were divided into 5 groups; Normal $=$ no discrepancy between BMIPP and flow tracer accumulation, Mild = mildly decreased BMIPP accumulation relative to flow tracer accumulation, Moderate $=$ moderately decreased BMIPP accumulation, Severe $=$ severely decreased BMIPP accumulation, and None $=$ no myocardial BMIPP accumulation despite apparently normal flow tracer accumulation. Double circle, homozygous for the C478 T substitution; closed circle, heterozygous for the C-478 T substitution; shaded triangle, apical type of hypertrophic cardiomyopathy.

saline (PBS) containing $9 \mathrm{mmol} / \mathrm{L}$ EDTA and MNCs were diluted to $1 \times 10^{7} / \mathrm{ml}$ with plain PBS. Aliquots of PRP or MNCs $(100 \mu \mathrm{l})$ were incubated for $30 \mathrm{~min}$ at $4{ }^{\circ} \mathrm{C}$ with $10 \mu \mathrm{l}$ of a fluorescein isothiocyanate (FITC)-labeled anti-CD36 monoclonal antibody, (OKM5; Ortho Diagnostic Systems, Raritan, NJ, USA), and then washed 3 times with PBS containing $9 \mathrm{mmol} / \mathrm{L}$ EDTA or plain PBS. Flow cytometric analysis was performed using a Cytron flow cytometer (Ortho) with an argon laser $(488 \mathrm{~nm})$. A FITC-labeled mouse $F\left(a b^{\prime}\right)_{2}$ fragment was used as the negative control for platelets and monocytes not incubated with OKM5. The level of CD36 expression was determined as the percentage of FITC-OKM5 positive platelets relative to all counted platelets.

\section{Detection of C-478 T Substitution}

Total cellular mRNA was obtained from platelets and monocytes by the method described by Chomczynski and Sacchi! ${ }^{2}$ As a C T transition at nucleotide (nt) 478 (C-478

T), which was found in 4 out of 5 patients with type II CD36 deficiency, has been reported, we analyzed this substitution using the method described by Kashiwagi et al! ${ }^{3}$ 
Table 1 Expression Level of Platelet CD36, Presence of a Point Mutation in CD36 cDNA, and BMIPP Uptake

\begin{tabular}{|c|c|c|c|c|c|c|c|c|}
\hline Case & Age & Sex & Diag & $I V S d$ & Ratio & $C D 36$ & Pro90 Ser & Mismatch \\
\hline 1. K.S. & 63 & $M$ & $H C M$ & 1.6 & 1.33 & 0.3 & $(+) *$ & None \\
\hline 2. J.N. & 36 & $M$ & $H C M$ & 1.8 & 1.80 & 0.2 & $(+)$ & Severe \\
\hline 3. J.S. & 60 & $M$ & $H C M$ & 1.2 & 1.20 & 0.3 & $(+)$ & Severe \\
\hline 4. K.H. & 57 & $F$ & $H C M$ & 1.8 & 1.50 & 0.4 & $(+)$ & Severe \\
\hline 5. H.E. & 68 & $M$ & $H C M$ & 1.2 & 1.38 & 0.4 & $(-) \min$ & Moderate \\
\hline 6. K.M. & 32 & $F$ & $H C M \$$ & 1.2 & 1.20 & 0.5 & $(-)$ & Moderate \\
\hline 7. T.S. & 82 & $M$ & $H C M$ & 1.3 & 1.30 & 0.9 & $(+)$ & Moderate \\
\hline 8. N.T. & 65 & $M$ & $H C M$ & 1.4 & 1.27 & 0.9 & $(-)$ & Mild \\
\hline 9. N.T. & 39 & $M$ & $H C M$ & 3.4 & 3.09 & 1.1 & $(-)$ & Moderate \\
\hline 10. Y.N. & 40 & $M$ & $H C M$ & 2.1 & 1.91 & 1.2 & $(-)$ & Severe \\
\hline 11. T.O. & 32 & $M$ & $H C M \$$ & 1.2 & 1.20 & 1.2 & $(+)$ & Moderate \\
\hline 12. M.Y. & 61 & $F$ & $H C M$ & 2.0 & 1.82 & 1.7 & $(+)$ & Moderate \\
\hline 13. S.H. & 43 & $M$ & $H C M$ & 1.4 & 1.18 & 25.4 & $(+)$ & Moderate \\
\hline 14. T.S. & 54 & $F$ & $H C M \$$ & 1.6 & 1.23 & 38.3 & $(-)$ & Normal \\
\hline 15. T.W. & 62 & $M$ & $H C M$ & 1.8 & 1.29 & 38.7 & $(-)$ & Moderate \\
\hline 16. K.S. & 43 & $M$ & $H C M$ & 2.0 & 1.67 & 42.6 & $(-)$ & Moderate \\
\hline 17. I.T. & 39 & $M$ & $H C M$ & 1.5 & 1.50 & 43.6 & $(-)$ & Moderate \\
\hline 18. T.K. & 20 & $M$ & $H C M \$$ & 2.1 & 1.75 & 45.5 & $(-)$ & Severe \\
\hline 19. K.F. & 37 & $M$ & $H C M$ & 2.5 & 2.27 & 46.5 & $(-)$ & Severe \\
\hline 20. M.Y. & 66 & $M$ & $H C M$ & 1.4 & 1.20 & 47.6 & $(-)$ & Moderate \\
\hline 21.S.S. & 29 & $M$ & $H C M \$$ & 2.0 & 2.22 & 48.0 & $(-)$ & Severe \\
\hline 22. F.M. & 61 & $F$ & $H C M$ & 1.6 & 1.23 & 59.6 & $(-)$ & Severe \\
\hline 23. Y.T. & 46 & $M$ & $H C M$ & 1.7 & 1.21 & 60.4 & $(-)$ & Mild \\
\hline 24. S.T. & 44 & $F$ & $H C M \$$ & 1.9 & 2.11 & 69.0 & $(-)$ & Moderate \\
\hline 25. H.O. & 26 & $M$ & $H C M \$$ & 1.6 & 2.00 & 73.5 & $(-)$ & Moderate \\
\hline 26. S.F. & 46 & $M$ & $H C M$ & 1.2 & 1.20 & 76.5 & $(-)$ & Mild \\
\hline 27. K.I. & 47 & $M$ & $H C M$ & 1.4 & 1.40 & 76.7 & $(-)$ & Severe \\
\hline 28. Y.O. & 41 & $M$ & $H C M$ & 1.3 & 1.30 & 77.0 & $(-)$ & Mild \\
\hline 29. H.K. & 60 & $M$ & $H C M$ & 1.6 & 1.60 & 77.1 & $(-)$ & Mild \\
\hline 30. Y.H. & 71 & $F$ & $H C M$ & 1.4 & 1.27 & 82.5 & $N D$ & Mild \\
\hline 31. T.K. & 57 & $M$ & $H C M$ & 2.3 & 1.77 & 83.4 & $(+)$ & Moderate \\
\hline 32. S.T. & 60 & $M$ & HCM\# & 1.6 & 1.45 & 86.3 & $N D$ & Normal \\
\hline 33. A.T. & 60 & $F$ & $H C M \$$ & 1.5 & 3.00 & 86.4 & $(-)$ & Moderate \\
\hline 34. Y.S. & 54 & $M$ & $H C M$ & 1.9 & 1.90 & 87.6 & $(+)$ & Severe \\
\hline 35. S.K. & 52 & $F$ & $H C M \$$ & 2.3 & 2.88 & 89.7 & $(-)$ & Mild \\
\hline 36. Z.K. & 59 & $M$ & $H C M$ & 1.8 & 1.64 & 90.0 & $N D$ & Moderate \\
\hline 37. T.I. & 58 & $M$ & HCM\# & 1.5 & 1.25 & 91.7 & $N D$ & Mild \\
\hline 38. M.T. & 38 & $M$ & $H C M$ & 1.8 & 1.38 & 92.1 & $N D$ & Mild \\
\hline 39. Y.K. & 55 & $M$ & HCM\# & 1.5 & 1.36 & 92.5 & $(-)$ & Normal \\
\hline 40. K.K. & 50 & $M$ & $H C M$ & 1.3 & 1.63 & 93.3 & $(-)$ & Moderate \\
\hline 41. I.O. & 62 & $M$ & $H C M$ & 2.3 & 1.77 & 93.3 & $(-)$ & Moderate \\
\hline 42. O.I. & 53 & $M$ & HCM\# & 1.5 & 1.00 & 94.2 & $N D$ & Mild \\
\hline 43. Y.H. & 53 & $F$ & $H C M$ & 1.6 & 2.00 & 94.2 & $N D$ & Mild \\
\hline 44. S.I. & 57 & $M$ & $H C M$ & 1.4 & 1.40 & 94.2 & $N D$ & Mild \\
\hline 45. K.K. & 52 & $M$ & $H C M$ & 1.5 & 1.25 & 94.5 & $N D$ & Mild \\
\hline 46. S.Y. & 48 & $M$ & $H C M$ & 2.0 & 1.82 & 94.9 & $(-)$ & Moderate \\
\hline 47. S.Y. & 69 & $M$ & $H C M \$$ & 1.8 & 1.64 & 96.0 & $(-)$ & Severe \\
\hline 48. К.O. & 52 & $M$ & $H C M$ & 1.3 & 1.30 & 96.2 & $N D$ & Mild \\
\hline 49. T.T. & 54 & $M$ & HCM\# & 1.3 & 1.30 & 96.6 & $N D$ & Mild \\
\hline 50. J.I. & 45 & $M$ & $H C M$ & 1.9 & 1.58 & 96.8 & $(-) \min$ & Normal \\
\hline 51. T.I. & 51 & $M$ & HCM\# & 1.2 & 0.92 & 97.0 & $N D$ & Normal \\
\hline 52. Y.K. & 64 & $M$ & $H C M$ & 1.4 & 1.40 & 97.7 & $N D$ & Mild \\
\hline 53. K.S. & 62 & $M$ & $H C M$ & 2.0 & 2.22 & 98.4 & $N D$ & Moderate \\
\hline 54. H.D. & 59 & $M$ & $H C M$ & 1.4 & 1.75 & 98.5 & $N D$ & Normal \\
\hline 55. T.Y. & 61 & $F$ & $H C M$ & 1.4 & 1.40 & 99.2 & $N D$ & Mild \\
\hline
\end{tabular}

IVSd, interventricular septal thickness; CD36, expression level of platelet CD36; Pro90 Ser, presence of the C-478 T substitution; Mismatch, the discrepancy between myocardial BMIPP accumulation and flow tracer accumulation as described in Fig 3; HCM\$, family history of hypertrophic cardiomyopathy; HCM\#, apical hypertrophy type of hypertrophic cardiomyopathy; (+)*, homozygote for of the C-478 T substitution; ND, not determined.

\section{Results}

Table 1 shows the expression of CD36 by platelets, the presence of the $\mathrm{C}-478$ T substitution in CD36 cDNA, and the arbitrary score of myocardial BMIPP uptake. Ten patients had a family history of HCM (cases $6,11,14,18$, $21,24,25,33,35$, and 47). Two patients had received myotomy and/or myectomy because of severe left ventricu- lar outflow tract obstruction (cases 24 and 41). Six patients showed apical hypertrophy type HCM (cases $32,37,39$, 42, 49, and 51).

Case 1 showed negligible CD36 expression on both his platelets and monocytes ( $0.3 \%$ and $1.4 \%$, respectively), indicating that he had type I CD36 deficiency as reported by Yamamoto et al! ${ }^{14}$ Analysis of CD36 cDNA revealed that case 1 was homozygous for the C-478 T substitution (Fig 2, lane 2). BMIPP scintigraphy revealed no myocardial BMIPP accumulation, although myocardial perfusion and hepatic BMIPP accumulation seemed to be normal, as reported previously6

In 11 patients (cases 2-12), platelets showed negligible expression of CD36. However, monocytes expressed CD36, indicating that these patients had type II CD36 deficiency! ${ }^{14}$ Among these 11 patients, 6 (cases 2-4, 7, 11, and 12) were found to be heterozygous for the $\mathrm{C}-478 \mathrm{~T}$ substitution (Fig 2, lanes 3,5, and 8). Moderately to severely decreased myocardial BMIPP uptake was documented in 10 patients, but myocardial BMIPP scintigraphy for case 8 showed mildly decreased myocardial BMIPP accumulation.

In 9 patients, CD36 was expressed by less than $50 \%$ of their platelets in spite of the normal expression of monocyte CD36 (cases 13-21). Re-evaluation of CD36 expression level in their platelets revealed almost the same values. Myocardial scintigraphy revealed severely decreased BMIPP accumulation in 2 (cases 18 and 21), moderately decreased BMIPP accumulation in 6 (cases 13, 15, 16, 17, 19, and 20), and apparently normal BMIPP accumulation in 1 (case 14). Among these patients, 1 showed the C-478

T substitution in CD36 cDNA (case 13, Fig 2, lane 4).

Two other patients (cases 31 and 34) also showed heterozygosity for the C-478 T substitution, although apparently normal expression of CD36 was observed on their platelets and monocytes (Fig 2, lanes 6 and 7). Myocardial scintigraphy revealed severely decreased BMIPP accumulation in case 34, and moderately decreased BMIPP accumulation in case 31 .

On the other hand, myocardial scintigraphy performed in 20 patients (cases 36-55) with platelet CD36 expression above $90 \%$ revealed no severe or moderate decrease in myocardial BMIPP accumulation, except for 6 patients (cases 36, 40, 41, 46, 47 and 53).

Fig 3 illustrates the relationship between the expression of CD36 by platelets and the myocardial BMIPP accumulation score. This figure shows that 23 out of $55 \mathrm{HCM}$ patients $(41.8 \%)$ had negligible or reduced $(>50 \%)$ levels of CD36 expression on platelets, or had a point mutation of CD36 cDNA (closed circles), and arbitrary myocardial BMIPP accumulation score revealed impaired LCFA accumulation in $95.5 \%$ of these patients $(21 / 23)$. On the other hand, 14 of the 20 patients $(70 \%)$ with CD36 by more than $90 \%$ of platelets showed no severe impairment of myocardial LCFA accumulation. In addition, patients with apical type HCM (shaded triangles) showed normal CD36 molecule expression and no severe impairment of myocardial BMIPP accumulation in this study.

\section{Discussion}

LCFAs are taken up by myocardial cells depending on the regional myocardial blood flow. Abnormal myocardial LCFA uptake has been demonstrated in some patients with HCM by BMIPP scintigraphy? Indeed, in this study, 33 
HCM patients (60\%) demonstrated obviously abnormal myocardial BMIPP accumulation (a score of moderate or more). However, the impaired metabolic pathway site(s) involved in these patients have not been investigated. BMIPP is thought to have an extraction fraction similar to native LCFAs, but does not undergo $\beta$-oxidation from its branching methyl group at the $\beta$-position.$^{15}$ Therefore, impaired myocardial BMIPP uptake with apparently normal myocardial perfusion may be considered to indicate an abnormality of LCFA metabolism before $\beta$-oxidation, such as the first step in LCFA metabolism, ie, LCFA uptake mechanism at the myocardial cell membrane. We recently proposed that myocardium took up LCFA via CD36, $, 6,8$ and also found a high prevalence of CD36 deficiency in HCM6 However, in that preliminary study, the number of enrolled patients was small, and distinct analyses of the CD36 molecule abnormality and myocardial LCFA accumulation were not achieved. Therefore, we analyzed the characteristics of the CD36 molecule and investigated the relationship between the abnormality of the CD36 molecule and myocardial BMIPP accumulation in $55 \mathrm{HCM}$ patients.

In this study, 12 patients (cases 1-12) showed negligible platelet CD36 expression and 9patients (cases 13-21) showed markedly decreased CD36 expression (below $50 \%$ ). Additionally, 2 patients were found to be heterozygous for the C-478 T substitution despite no significant reduction in CD36 expression. If all these 23 patients were assumed to have abnormalities of $\mathrm{CD} 36$, the prevalence of patients with abnormal CD36 molecules in HCM was $41.8 \%$ (23/55), which was similar to our previous report $(37.9 \%)$ ? Among these 55 HCM patients, 6 (cases 32, 37, $39,42,49$, and 51) demonstrated apical hypertrophic HCM. The expression level of CD36 on their platelets was not reduced $(86.3 \%, 91.7 \%, 92.5 \%, 94.2 \%, 96.6 \%$, and $97.0 \%$, respectively), and no significant abnormality of myocardial LCFA accumulation was demonstrated in their BMIPP scintigraphy. Accordingly, the etiology of HCM with asymmetrical septal hypertrophy (ASH) and with apical hypertrophy might be different. Possible mechanisms for the etiology of HCM with ASH have been discussed previously 6

The impairment of myocardial BMIPP accumulation was significant in HCM patients with a low level of CD36 expression or whose CD36 cDNA showed a point mutation at $n t 478$ (C-478 T substitution) $(21 / 23,95.5 \%)$. Thus, impairment of myocardial BMIPP uptake in some HCM patients may be associated with abnormalities of CD36. However, some patients showed no significant impairment of myocardial BMIPP accumulation despite decreased expression of CD36 (cases 8 and 14). Furthermore, impaired myocardial BMIPP accumulation was observed in some patients whose expression of CD36 was not reduced (cases 36, 40, 41, 46, 47, and 53).

In this study, we screened the CD36 molecular abnormality by measuring the expression level of the CD36 molecule on platelets and monocytes. Unfortunately, analysis of CD36 cDNA was not performed in some of the patients, especially those who showed high levels of platelet CD36 expression. CD36 cDNA analysis revealed that 7 patients, who showed negligible or significantly decreased levels of CD36 expression on their platelets, were heterozygous for the C-478 T substitution (cases 2, 3, 4, 7, 11, 12, and 13). Although 2 patients (cases 31 and 34 ) were also heterozygous for this point mutation, a relatively high level of
CD36 expression was observed on their platelets. The reason for this is unknown. In this study, CD36 cDNA analysis was only performed at nt 478 (C-478 T). The C-478 $\mathrm{T}$ substitution was not found in 5 patients (cases 5, 6, 8, 9, and 10) despite negligible platelet expression of CD36 or in 8 patients (cases 14-21) with CD36 expression on less than $50 \%$ of their platelets. Thus, the genetic abnormalities of these patients are unknown at present. In addition, CD36 deficiency was only screened by CD36 expression on platelets and monocytes as a substitute for assessing CD36 molecules in the myocardium. Hence, the low expression of CD36 on platelets and monocytes, especially when the genetic abnormality is not yet known, might not be representative of myocardial disease. Thus, the limitations of this study include the use of platelets and monocytes rather than direct measurement of CD36 molecules in the myocardium. Future work should be carried out to address these issues.

In summary, no myocardial BMIPP uptake was observed in $1 \mathrm{HCM}$ patient with type I CD36 deficiency (homozygous for the C-478 T substitution), and severely decreased myocardial BMIPP uptake was observed more frequently in HCM patients with negligible platelet expression of CD36 and/or heterozygous for the C-478 T substitution in CD36 cDNA. On the other hand, most HCM patients, having platelets with apparently normal levels of CD36 expression, showed no significant impairment of myocardial BMIPP accumulation. Hitherto, an abnormal myocardial LCFA metabolism seen in some patients with HCM has been unclear, and has been discussed from the view of abnormal mitochondrial function or ischemia. The present study may suggest a possible explanation for impaired myocardial LCFA metabolism seen in HCM. In this study, we showed a high frequency of abnormal CD36 expression in patients with HCM whose myocardial BMIPP scintigraphy also suggested an impaired myocardial LCFA metabolism. Thus, abnormalities of the CD36 molecules may be related to the pathogenesis of some types of HCM.

\section{Acknowledgments}

We are indebted to Drs Yasushi Kitaura and Hirofumi Deguchi for their clinical support. We are also indebted to Drs Michihiro Suwa, Yoshiaki Otake, Takahide Ito, and Ayaka Kobashi for performing the echocardiographic and/or myocardial scintigraphic studies.

\section{References}

1. Abumurad N, El-Maghrabi M, Amri E-Z, Lopez E, Grimaldi P. Cloning of a rat adipocyte membrane protein implicated in binding or transport of long-chain fatty acids that is induced during preadipocyte differentiation. J Biol Chem 1993; 268: 17665-17668

2. Tanaka $\mathrm{T}$, Kawamura K: Isolation of myocardial membrane longchain fatty acid-binding protein: homology with a rat membrane protein implicated in the binding or transport of long-chain fatty acids. $J$ Mol Cell Cardiol 1995; 27: 1613-1622

3. Ikeda H, Mitani T, Ohnuma M, et al: A new platelet-specific antigen, $\mathrm{Nak}^{\mathrm{a}}$, involved in the refractoriness of HLA-matched platelet transfusion. Vox Sang 1989; 57: 213-217

4. Yamamoto N, Ikeda H, Tandon N, et al: A platelet membrane glycoprotein (GP) deficiency in healthy blood donors: Nak(a-) platelet lack detectable GP IV (CD36). Blood 1990; 76: 1698-1703

5. Yamamoto N, Akamatsu N, Yamazaki H, Tanoue K: Normal aggregations of glycoprotein IV (CD36)-deficient platelets from seven healthy Japanese donors. Br J Haematol 1992; 81: 86-92

6. Tanaka T, Sohmiya K, Kawamura K: Is CD36 deficiency an etiology of hereditary hypertrophic cardiomyopathy? J Mol Cell Cardiol 1997; 29: 121-127

7. Inoue $\mathrm{F}$, Hashimoto $\mathrm{T}$, Nishida $\mathrm{Y}$, Dohi $\mathrm{K}$, Matsushima A, 
Sakakibara H, et al: Absence of myocardial 123I-BMIPP uptake in the presence of a normal coronary angiogram and normokinetics on a left ventriculogram. Jpn Circ J 1997; 61: 263-267

8. Tanaka T, Okamoto F, Sohmiya K, Kawamura K: Lack of myocardial iodine-123 15-(p-iodiphenyl)-3-R, S-methylpentadecanoic acid (BMIPP) uptake and CD36 abnormality - CD36 deficiency and hypertrophic cardiomyopathy. Jpn Circ J 1997; 61: 724-725-

9. Takeishi Y, Chiba J, Abe S, Tonooka I, Komatani A, Tomoike H: Heterogenous myocardial distribution of iodine-123 15-( $p$ iodiphenyl)-3-R,S-methylpentadecanoic acid (BMIPP) in patients with hypertrophic cardiomyopathy. Eur J Nucl Med 1992; 19: $775-782$

10. Kusaka Y, Tanaka T, Okamoto F, Terasaki F, Matsunaga Y, Miyazaki H, et al: Effect of sulfo-N-succinimidyl palmitate on the rat heart: myocardial long-chain fatty acid uptake and cardiac hypertrophy. J Mol Cell Cardiol 1995; 27: 1605-1612

11. Rupp H, Elimban V, Dhalla N: Modification of subcellular organelles in pressure-overloaded heart by etomoxir, a carnitine palmitoyltransferase I inhibitor. FASEB J 1992; 6: 2349-2353

12. Chomczynski P, Sacchi N: Single-step method of RNA isolation by acid guanidinium thiocyanate-phenol-chloroform extraction. Anal Biochem 1987; 162: 156-159

13. Kashiwagi $\mathrm{H}$, Honda $\mathrm{S}$, Tomiyama $\mathrm{Y}$, et al: A novel polymorphism in glycoprotein IV (replacement of proline-90 by serine) predominate in subjects with platelet GPIV deficiency. Thromb Haemost 1993; 69: 481-484

14. Yamamoto N, Akamatsu N, Sakuraba H, Yamazaki H, Tanoue K: Platelet glycoprotein IV (CD36) deficiency is associated with the absence (type I) or the presence (type II) of glycoprotein IV on monocytes. Blood 1994; 83: 392-397

15. Knapp FJ, Ambrose KR, Goodman MM: New radioiodinated methyl-branched fatty acids for cardiac studies. Eur J Nucl Med 1986; 12: s39-s44 